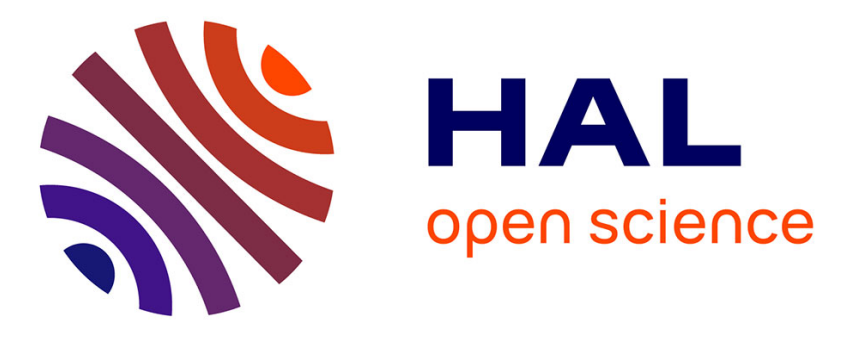

\title{
Forecasting of renewable energy balance on Medium Term.
}

Otilia Elena Dragomir, Florin Dragomir, Rafael Gouriveau, Eugénia Minca

\section{To cite this version:}

Otilia Elena Dragomir, Florin Dragomir, Rafael Gouriveau, Eugénia Minca. Forecasting of renewable energy balance on Medium Term.. Conference "Large Scale Systems, Theory and Applications", LSS'10., Jul 2010, Villeneuve d'Ascq, France. pp.1-6. hal-00544703

\section{HAL Id: hal-00544703 https://hal.science/hal-00544703}

Submitted on 8 Dec 2010

HAL is a multi-disciplinary open access archive for the deposit and dissemination of scientific research documents, whether they are published or not. The documents may come from teaching and research institutions in France or abroad, or from public or private research centers.
L'archive ouverte pluridisciplinaire HAL, est destinée au dépôt et à la diffusion de documents scientifiques de niveau recherche, publiés ou non, émanant des établissements d'enseignement et de recherche français ou étrangers, des laboratoires publics ou privés. 


\title{
Forecasting of Renewable Energy Balance on Medium Term
}

\author{
Otilia Elena Dragomir*, Florin Dragomir*, Rafael Gouriveau**, Eugenia Minca* \\ * Valahia University of Târgoviste, Electrical Engineering Faculty, Automation and Information Department, Unirii Avenue \\ 18-20,Târgoviste,_Romania, (e-mail: \{drg_otilia,drg_florin,_minca\}@yahoo.com). \\ ** Ecole Nationale Supérieure de Micromécanique et des Microtechniques, Laboratoire d'Automatique de Besançon, 25000 \\ Besançon, France, e-mail: rgourive@ens2m.fr
}

\begin{abstract}
The general purpose of the paper is to explore the way of performing renewable energy balance predictions prognostics so that energy market actors can act consequently. Different aspects of forecasting are discussed to point out pragmatic challenges of this approach. An illustration, with real monitored data, based on a neuro-fuzzy predictor is given. The architecture of the artificial intelligence technique used for forecasting is modified in order to obtain accurate estimations for medium term.
\end{abstract}

Keywords: forecasting, neuro- fuzzy, renewable energy balance, photovoltaic system.

\section{INTRODUCTION}

Nowadays there are huge ranges of energy market participants, bidding strategies are more and more complex, and a number of financial derivatives have been developed. Commercial success depends on the ability to submit competitive predictions relative to energy balance trends (difference between the electricity produced and consumed DPcg) (Dragomir F. et al., 2009). Thus, it seems convenient to "anticipate" a phenomenon in order to act consequently and resort to protective actions.

In particular, the different time horizons required by the electricity markets (e.g., day-ahead, hour-ahead) can be respectively associated to short term (STLF) (Hippert et al., 2001), medium term (MTLF) or with long term forecasting (LTLF) (Desons et al., 1997). For short term forecasting the information is sampled on an hourly (or half hourly) basis, or even a daily basis (for load peak prediction) so is defined as varying from a few minutes up to a few weeks ahead. This type of prediction is important because the national grid requires DPcg values at any moment in the day. Traditionally, hourly forecasts with a lead time between one hour and seven days are required for the scheduling and control of power systems. The medium term forecasting in energy field covers the horizon from one month up to a few years ahead and finally, the long term horizon considers load peaks and consumed energy, on a yearly basis, for several years ahead. For example, long-term forecasting is relevant for the planning of new electricity utilities, and inaccurate predictions have important financial costs. For this particular forecasting case is a need for accurate prediction.

From the perspective of the system operators and regulatory agencies, the medium term forecasting is a source of primary information for the safe and reliable operation of the system. For producers also, these ones are a basic tool for determining the optimal utilization of generators and power stations, as some facilities are more efficient than others.
Despite the many publications and models of load forecasting that have been developed in the last few decades, few amongst those have dealt with the medium term forecasting.

This paper deals with this type of forecasting. Precisely, it is based on neuro - fuzzy networks in order to estimate the DPcg evolution for a medium time horizon. This artificial intelligence tool is applied is illustrated on a data based obtain from an experimental photovoltaic amphitheatre of minimum dimension $(0.4 \mathrm{kV} / 10 \mathrm{~kW})$, located in the eastcentre region of Romania, more precisely in the city of Targoviste (ICOP DEMO, 1998).

In this context, the paper is organized in two parts. First of all, the forecasting framework is delimited, starting with the definition, metrics, approaches and tools. In the second section the chose of a neuro - fuzzy tool is argued using the existed approaches and the results obtained by different authors. A new ANFIS (Adaptive Neuro Fuzzy Inference System) architecture is proposed, illustrated and discussed.

Efforts improving the photovoltaic (PV) module performance in different climates have been increasing over the years. With new materials entering the market place, this task is gaining further in importance. Using standard test condition efficiency in the design stage is not enough appropriate. It is therefore imperative a true understanding of the reasons for varying performance before the design stage. Additionally, the accurate separation and quantification of different effects influencing the performance would be valuable asset to the PV community.

\section{ENERRGY FORECASTING}

\subsection{Forecasting concepts}

Considering the benefits that forecasting may bring to the security, economics and resource management fields, the scientific community is now beginning to take some interest 
in this area. The control of the performance prediction represents the premise of a good global performance.

In literature, the forecasting is called also prediction or prognosis and this reveals that there is no consensual acceptation of term: (Rytter, 1993), (Lin et al., 2003), (ISO, 2004), (Dragomir O. et al., 2007). Due to these facts, in this article the forecasting will be associated with the notion of prediction and will determine the future state of the analyzed system the closest possible to the future real state of the system.

\subsection{Forecasting measures}

There is no general agreement as to an appropriate and acceptable set of metrics that can be employed effectively to assess the performance of the prediction. The performance measures will evolve with time as more data are available, and the measures are expected to improve over time [9]. Beyond this aspect, very important is the error value determination (where the error is defined as the difference between real and estimated value of the analysed system parameter) (Vachtsevanos et al., 2006). Therefore, given the same error size corresponding to a certain magnitude of deviation, it is in most situations preferable to have a positive bias (early prediction), rather than a negative one. Of course, one needs to define two different boundaries for the maximum acceptable late prediction and the maximum acceptable early one. Any prediction outside of it will be considered either a false positive or a false negative (Goebel et al., 2005).

Measurement of forecasting performance quality is highly dependent on how rigidly the criteria are specified, and are is largely dependent on user-specifications (Bonissone et al., 2007). So a critical point at this level is what criterion can be used to measure the accuracy of the predicted results.

Accuracy measures the closeness of the predicted value to the actual value. It is highest when the predicted value is the same as the actual value and decreases when the predicted value deviates from the actual value so the sensitivity will be very low when the predicted value deviates too much.

Another metric, often use in a prediction performance measure is the precision. The precision implies how close the predictions are bunched or clustered together and is a measure of the narrowness of un interval in which the system falls. Precision is defined on the basis of the variance of the predicted results for many experiments It is high if the predicted values are clustered together around the actual value and it is low if the predicted values are scattered over the output range.

Confidence level of the forecasting indicates in percentage value, the degree of certitude of the future predicted value modes or of the estimation made. An average of the width of the confidence intervals of the prediction algorithm in the precision definition is often use because a narrower confidence interval gives higher precision.
Applying different methods for forecasting DPcg, in our studied case, the final objective is the assurance of a certain level of error for a given prediction horizon (Fig. 1).

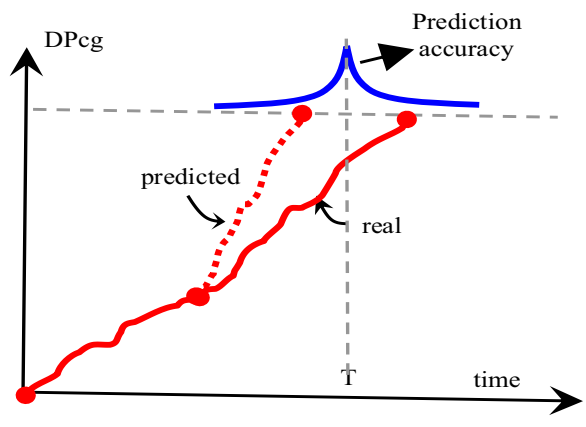

Fig. 1. Forecasting error measurements

\subsection{Forecasting approaches}

The literature has presented a great variety of methodologies for electricity forecasting. Contributions can be distinguished between statistical approaches and exponential smoothing approaches, between unvaried methods and methods with explanatory variables, between linear models and nonlinear models.

Earlier papers have developed both single-equation models and multiple-equation models with different equations for different hours of the day. The time dependence of hourly loads has been captured observation-driven models and parameter-driven models with unobserved components. Articles that deal with model based load forecasting include: (Cancelo et al., 2008), (Liu et al., 2006), (Soares et al., 2008), (Tyler et al., 2003) and (Tyler et al., 2006).

However, deregulated energy markets have presented new challenges to decision making, requiring more information which is dependent forecasting. Therefore, the corresponding development and maintenance efforts for dealing with hundreds of irregular data series, which need to be simultaneously forecasted for security and economical analyses, means that the parametric models are beyond practical consideration. The relationship between the electricity load and its exogenous factors is complex and nonlinear, making it quite difficult to represent using linear models, or even parametric nonlinear ones.

In recent years, papers have shown the potential of data driven forecasting approaches (Dragomir O. et al., 2009). Data-driven approaches derive directly from routinely monitored system operating data. So, in many applications, measured input/output data is the major source for a deeper understanding of the system degradation behavior. These rely on the assumption that the statistical characteristics of data are relatively unchanged unless a malfunctioning even occurs.

The strength of data-driven techniques is their ability to transform high-dimensional noisy data into lower dimensional information for diagnostic/prognostic decisions. 
Artificial intelligence (AI) techniques have been increasingly applied to systems forecasting and have shown improved performances over conventional approaches.

In practice however, it isn't easy to apply AI techniques due to the lack of efficient procedures to obtain training data and specific knowledge. So far, most of the applications in the literature just use experimental data for model training. Thus, data-driven approaches are highly-dependent on the quantity and quality of system operational data.

\subsection{Forecasting tools}

Actually the systems are very complexes and the conditioning parameters that influence system functioning are significant. Often their actions induce nonlinearities in system modelling process because can not be always precisely described. In these cases it is very difficult to determine any sort of model for prediction purposes.

Artificial Neural Networks (ANNs) are techniques use often for load forecasting. For short term forecasting horizons in particular, the neural networks have a good flexibility in capturing nonlinear interdependencies between the load and exogenous variables. However, the ANN models are complex and difficult to understand, and are often over-fitted. Indeed, their structure is sufficiently opaque that it is not clear why they should forecast as well as they do. As a result, the literature is still undecided as to their practical utility for electricity load forecasting. Articles that deal with ANN models, in particular for short term load forecasting horizon are (Alves da Silva et al., 2000), (Khotanzad et al., 1998), (Darbellay et al., 2000), (Metaxiotis et al., 2003), (Reis et al., 2005), and (Hippert et al., 2005).

Despite all publicized successes, neural network based load forecasting models have been designed relying on timeconsuming, empirical, and suboptimal procedures (Ferreira et al., 2007). The potential for applying neural networks to short term load forecasting depends strongly on the extraction of appropriate input variables. This requirement has often been neglected, and many proposals for building the neural network input space still use (linear) correlation analysis. Input selection procedures with the capacity to extract high order statistical information from the input-output data must be employed to fully exploit the ANN mapping capability.

The advantages and the drawbacks of AI forecasting techniques, precisely of ANN, leaded us to the choosing of nonlinear network (neuro-fuzzy systems - NFs) approximates as reference tool for our approach of medium term energy balance forecasting.

Neuro- fuzzy systems are a combination of ANNs and fuzzy sets and represent a powerful tool to model systems behaviour. The ANN is used to define the clustering in the solution space, which results in creation of fuzzy sets (Jang et al., 1997).

A particular architecture of neuro-fuzzy systems is represented by the adaptive neuro-fuzzy inference system (ANFIS) (Jang, 1993). ANFIS is a Sugeno-type fuzzy inference system in which the parameters associated with specific membership functions are computed using either a backpropagation gradient descent algorithm alone or in combination with a least squares method. It has been widely applied to random data sequences with highly irregular dynamics (Wang, 2003) e.g. forecasting non-periodic shortterm stock prices (Chiang et al., 2001).

The success of ANFIS is given by aspects like: the designated distributive inferences stored in the rule base, the effective learning algorithm for adapting the system's parameters or by the own learning ability to fit irregular or non-periodic time series. On the other hand, used in application alone to non-periodic short-term forecasting, ANFIS predictions make large residual errors due to high residual variance, consequently degrading prediction accuracy (Gourierou , 1997). It is very difficult to interpret for a non expert the fuzzy rules generated by ANFIS because of the form of consequents (linear combination of inputs).

\section{NEURO FUZZY ENERGY BALANCES FORECASTING}

The actual challenges consist in controlling the performance of the future state of the system for medium term. The proposed framework consider forecasting process as a aggregate function of past, present and future states. The third variable who appears in prediction function definition is the results of the necessity of taken into account of "known future" actions dues of precise moments in the week or in the year For example, it is expected a modification of the consumers behaviours due summer, winter holydays or weekend time. (Fig. 2)

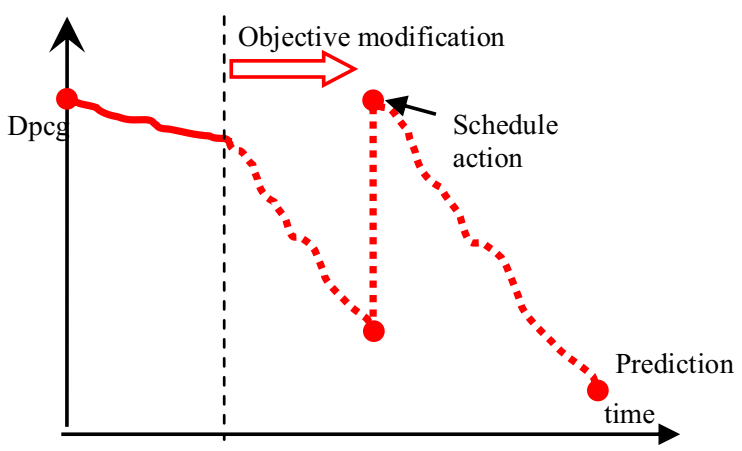

Fig. 2. Future states that influence prediction process

Most of the papers that use ANFIS for prediction consider the inputs as they exist in the benchmarks. In this paper ANFIS use a data base with 290 data points $\{\mathrm{y}(\mathrm{t}), \mathrm{u}(\mathrm{t})\}$, from $\mathrm{t}=1$ to $\mathrm{t}=296$. From the monitored plant, the Solar Amphitheatre [4], the DPcg is considered as output of the model - y(t) and the exterior temperature as input $-\mathrm{u}(\mathrm{t})$.

The choice of error measures to help comparing forecasting methods has been much discussed. A major part of them have been summarized by (Gooijer et al., 2006). Most authors consider that MAPE would be an adequate error measure if the loss function were linear (and linear in percentage, not in absolute error); however, recent studies and the experience of 
system operators indicates that the loss function in the load forecasting problem is clearly nonlinear, and that large errors may have disastrous consequences for a utility (Hobbs et al., 1999). Because of manner of the penalization of large errors this, measures based on root mean squared error was suggested in (Armstrong et al., 1995). Also, it is generally recognized that error measures should be easy to understand and closely related to the needs of the decision-makers. Some papers reported that the utilities would rather evaluate forecasting systems by the absolute errors produced, and this suggests that mean absolute errors could be useful (Mohammed et al., 1995).

In any case, error measures are only intended as summaries for the error distribution. This distribution is usually expected to be normal white noise in a forecasting problem, but it will probably not be so in a complex problem like load forecasting. No single error measure could possibly be enough to summarize it. The shape of the distribution should be suggested. Keeping the total error low, therefore, means keeping the model simple.

It is well known that goodness-of-fit statistics are not enough to predict the actual performance of a method. The implications and consequences of the choices made in design, implementation and validation of neuro-fuzzy architecture are very important. Often appears problems of overfitting or overparametrization (Steinherz et al., 2001).

Considering the metrics described above, we have observed that the ANFIS architecture, for two and three selected inputs, has satisfactory results for a short term prediction (Fig. 3)

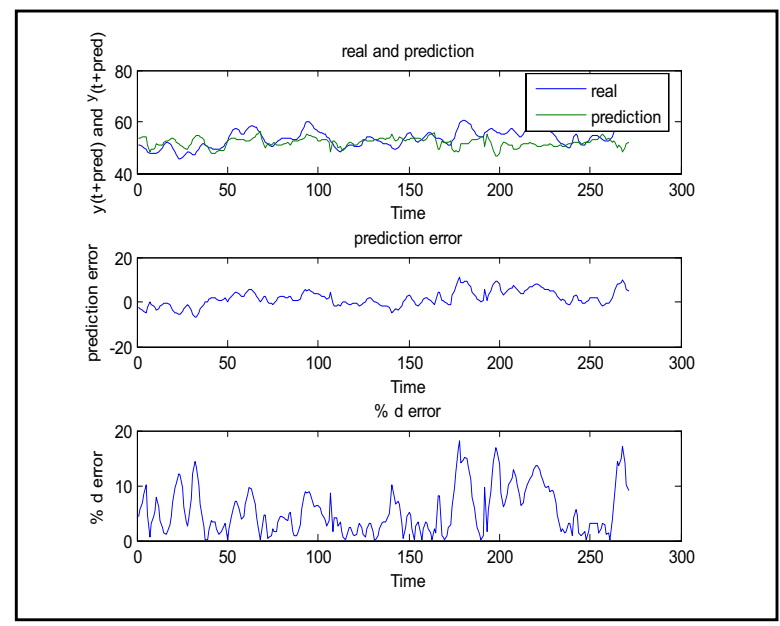

a)

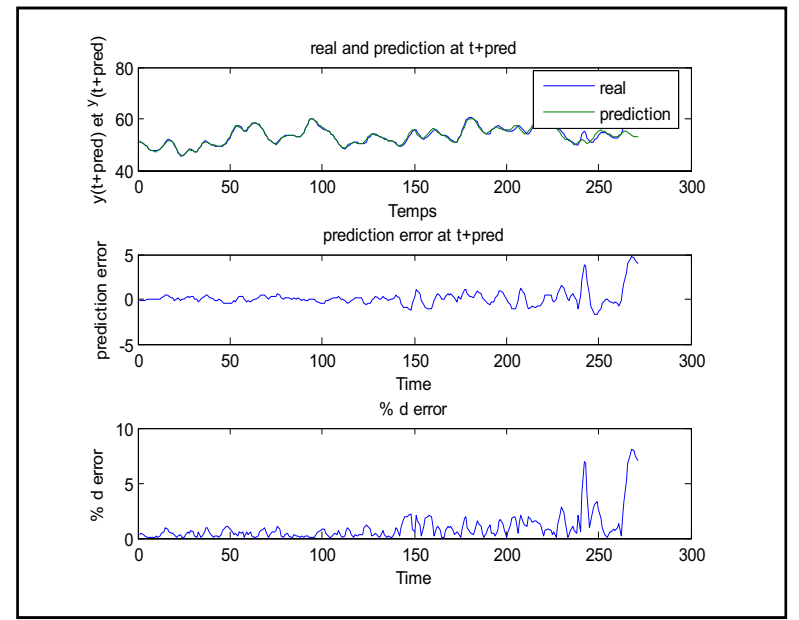

b)

Fig. 3. a) Prediction error for two inputs ANFIS for twenty steps ahead forecasting; b) Prediction error fortwo ANFIS linkt in series for twenty steps ahead forecasting

For medium and long term, the obtained errors became bigger and bigger and this affects the forecasting in terms of accuracy and confidence (Fig. 4).

The prediction results from Fig. 4 are obtained for an ANFIS with two selected inputs, for a time horizon equal with 1,10 and 20 .

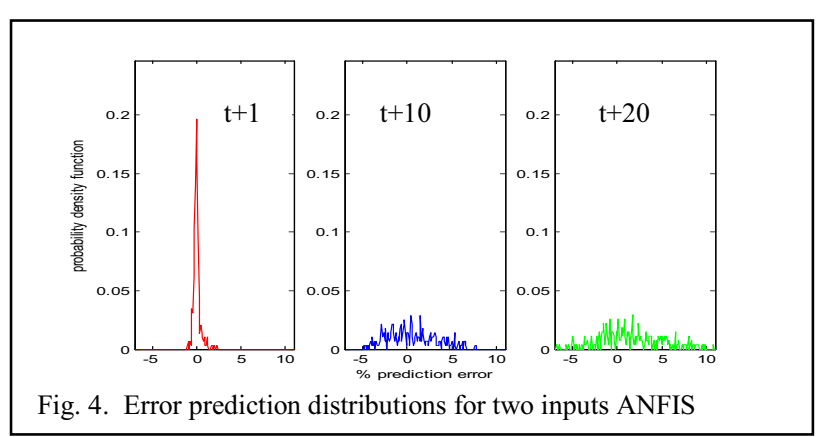

In order to obtain a good performances as result of implementing neuro-fuzzy tools in forecasting approaches, this article propose a novel architecture for ANFIS as an alternative for medium time error stabilization. This one link the ANFIS modules in series to penalize the growth tendency of error in time. The performance measured in terms of error became satisfactory a medium term (Fig. 5).

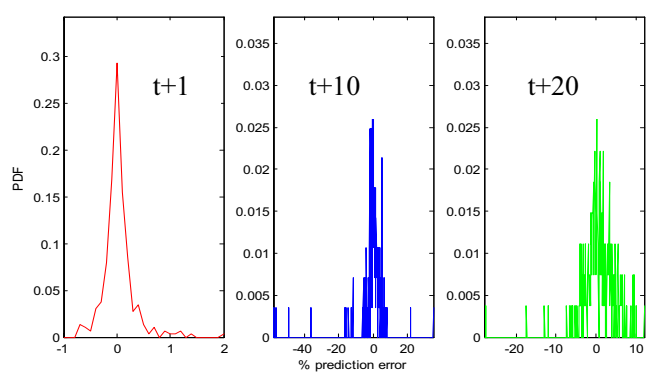

Fig. 5. Error prediction distributions for two ANFIS linkt in series 
The effect of taking into account the "future" for prediction, in other words the modification of the mission profile due to some extern intervention has also influence over error. (Fig. $6)$.
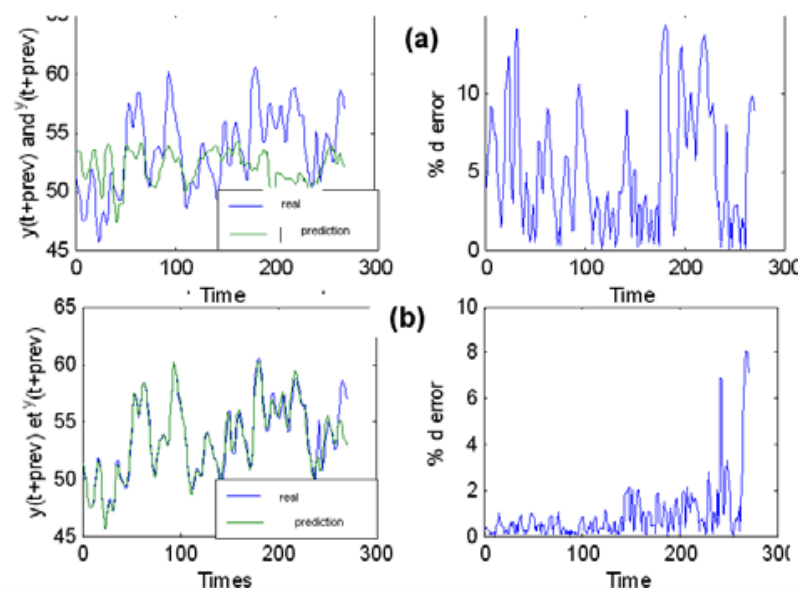

(b)

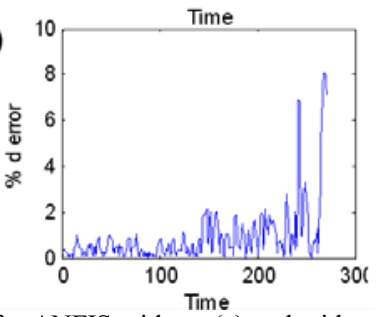

Fig. 6. Error prediction distributions for ANFIS without (a) and with (b) external interventions

\section{CONCLUSIONS AND WORK IN PROGRESS}

Controls of the performance prediction represent the premise of a good global forecasting performance. The existing approaches, methods, metrics and tools for load forecasting are discussed from different points of view. The identified problems and advantages create the context for proposing a new ANFIS architecture capable to ensure for the prediction stability in terms of errors magnitude for a medium term horizon. Also, the preplanned actions require the availability of assets under consideration for a specified time. The obtained confidence level at this point of time is a satisfactory one from the industrials point of view.

The work is still in progress and the developments are at present extended in three principal ways. First, the definition of new loss functions capable to penalize the errors above an acceptable tolerance level. Secondly, the application of other tools as technics for a global forecasting to be investigated. Finally, an amelioration of obtained predictive system in terms of interpretability.

\section{REFERENCES}

Alves da Silva A.P., L.S. Moulin (2000). Confidence intervals for neural network based short term load forecasting, IEEE Transactions on Power Systems, 15(4), 1191-1196.

Armstrong J.S., R. Fildes (1995). Correspondence on the selection of error measures for comparisons among forecasting methods, J. Forecast., 14, 67-71

Bonissone P., K. Goebel, "When will it break? A Hybrid Soft Computing Model to Predict Time-to-break Margins in paper machine's", In: Proc. SPIE 47th Annual Meeting, Int. Symp. Optical Science and Technology, 4787, 53-64

Cancelo J.R., A. Espasa, R. Grafe, (2008). Forecasting from one day to one week for the Spanish system operator, International Journal of Forecasting, 24, 588-602

Chiang L.H., E. Russel, R. Braatz (2001). Fault detection and diagnosis in industrial systems, Springer-Verlag, London.
Darbellay G., M. Slama (2000). Forecasting the short term demand for electricity: Do neural networks stand a better chance?, International Journal of Forecasting, 16, 71-83.

Desons J.Y., R.Ben Younes (1997). Prévision long terme a la réponse d'un stockage de chaleur sensible dans le sol, Elsevier Science.

Diebold F.X (1998). Elements of Forecasting, South-Western, Cincinnati , $\mathrm{OH}$

Dragomir F., S.S. Iliescu (2009). Fuzzy intelligent control for the low voltage electrical networks with distributed power generation from renewable energy resources, Scientific Bulletin of Electrical Engineering Faculty, Vol. 10, nr. 1 (10)

Dragomir O., R. Gouriveau, F. Dragomir, E. Minca (2009). Review of the prognosis problem in academicals and industrial area of interest, European Control Conference 2009- ECC'09, 23-26 August Budapest, Hungary

Dragomir O., R. Gouriveau, N. Zerhouni, F. Dragomir (2007). Framework for a distributed and hybrid prognostic system, 4th IFAC Conference on Management and Control of Production and Logistics, 27-30 Sept., Sibiu, Romania

European Research Programm ICOP-DEMO 4080/98, "Building Integration of Solar Technology" (http://dcem.valahia.ro)

Ferreira V.H., A.P.A. da Silva (2007). Toward estimating autonomous neural network-based electric load forecasters, IEEE Transactions on Power Systems, 22, 1554-1562.

Goebel Kai, P. Bonissone (2005). Prognostic information fusion for constant load systems, In: Proceedings of 7 th annual Conference on Fusion, 2, 1247-1255.

Gooijer J.G., R.J. Hyndman (2006). 25 years of time series forecasting, International Journal of Forecasting, 22 ,443- 473.

Gourierou C. (1997). ARCH Models and Financial Applications, Springer, NewYork.

Hippert H.S., C.E. Pedreira and R.C. Souza (2001). Neural networks for short-term load forecasting: a review and evaluation, IEEE Transactions on Power Systems, Vol.16, pp. 44-55

Hippert H.S., D.W. Bunn, R.W. Souza (2005). Large neural networks for electricity load forecasting: Are they overfitted?, International Journal of Forecasting, 21, 425-434,

Hobbs B.F., S. Jitprapaikulsarn, S. Konda, V. Chankong, K.A. Loparo, D.J. Maratukulam (1999). Analysis of the value for unit commitment of improved load forecasting, IEEE Trans. Power Systems, 14 (4), 13421348 .

ISO 13381-1 (2004). Surveillance et diagnostic des machines- Pronostic. Norme internationale- AFNOR

J.S.R Jang (1993). ANFIS: adaptive-network-based fuzzy inference systems, IEEE Trans. Systems, Man, Cybern., 23 (3), 665 -685.

Jang J.S.R, C.T. Suni, E. Mizutani (1997). Neuro-fuzzy and soft computing: a computational approach to learning and machine intelligence, Prentice Hal, New York.

Khotanzad A., R. Afkhami-Rohami, D. Maratukulam (1998). ANNSTLF Artificial Neural Network Short Term Load Forecaster - Generation, Three. IEEE Transactions on Power Systems, 13(4), 1413-1422.

Lin D., V. Makis (2003). Recursive filters for a partially observable system subject to random failure, Advances in Applied Probability, Vol. 35 , pp.207-227.

Liu J.M., R. Chen, L.M. Liu, J.L. Harris (2006). A semiparametric time series approach in modeling hourly electricity loads, Journal of Forecasting, 25, 537-559.

Metaxiotis K., A. Kagiannas, D. Askounis, J. Psarras (2003). Artificial intelligence in short term electric load forecasting: A state of the art survey for the researcher, Energy Conversion and Management, 44, 1525-1534,

Mohammed O., D. Park, R. Merchant, T. Dinh, C. Tong, A. Azeem, J. Farah, C. Drake (1995). Practical experiences with an adaptive neural network short-term load forecasting system, IEEE Trans. Power Systems, 10( 1), 254-265.

Park D.C., M.A. El-Sharkawi, R.J. Marks (1991). An adaptively trained neural network, IEEE Transactions on Neural Networks, 2, 334-345.

Reis A.J.R., A.P. Alves da Silva (2005). Feature Extraction Via MultiResolution Analysis for Short Term Load Forecasting, IEEE Transactions on Power Systems, 20(1), 189-198.

Rytter A. (1993). Vibration Based Inspection of Civil Engineering Structures, PhD Thesis.

Soares L.J., M.C. Medeiros (2008). Modeling and forecasting short-term electricity load: a comparison of methods with an application to Brazilian data, International Journal of Forecasting, 24, 630-644. 
Steinherz Henrique, Carlos Eduardo Pedreira, Reinaldo Castro Souza (2001) Neural Networks for Short- term Load Forecasting: A Review and Evaluation, IEEE Transactions on Power Systems, 16(1)

Taylor J.W., L.M. De Menezes, P.E. McSharry (2006). A comparison of univariate methods for forecasting electricity demand up to a day ahead, International Journal of Forecasting, 22, 1-16.

Taylor J.W., R. Buizza (2003). Using weather ensemble predictions in electricity demand forecasting, International Journal of Forecasting, $19,57-70$.

Vachtsevanos G., F.L. Lewis, M. Roen, A. Hess, R. Wu (2006). Intelligent Fault Diagnosis and Prognosis for Engineering System, John Wiley and Sons Inc, USA,

Wang J.S. (2003). An efficient recurrent neuro-fuzzy system for identification and control of dynamic systems, IEEE Internat. Conf. Systems, Man, and Cybernetics. 